\title{
HEVC Information-Hiding Algorithm Based on Intra-Prediction and Matrix Coding
}

\author{
Yong Liu, School of Mathematics and Big Data, Anhui University of Science and Technology, China \\ Dawen Xu, School of Electronics and Information Engineering, Ningbo University of Technology, China
}

\begin{abstract}
Aiming at the problem that the data-hiding algorithm of high efficiency video coding (HEVC) has great influence on the video bit rate and visual quality, an information-hiding algorithm based on intra-prediction mode and matrix coding is proposed. Firstly, eight prediction modes are selected from $4 \times 4$ luminance blocks in I frame to embed the hidden information. Then, the least significant bit (LSB) algorithm is used to modulate the LSB of the last prediction mode. Finally, the modulated luminance block is re-encoded to embed four bits secret information. Experimental results show that the algorithm improves the embedding capacity, guarantees the subjective and objective quality of the video, and the bit rate increases by $1.14 \%$ on average.
\end{abstract}

\section{KEYWORDS}

HEVC, Information Hiding, Intra Prediction, Least Significant Bit, Matrix Coding

\section{INTRODUCTION}

With the development of digital multimedia technology, more multimedia resources are widely used (Mohanarathinam, A., Kamalraj, S., \& G, K. D., 2019). However, in the process of video transmission and storage, some important information may be illegally tampered, misappropriated and attacked. Information hiding is a technology to embed data into media content, which mainly including steganography and digital watermarking. The technology has been used in many application scenarios, such as ownership declaration protection, privacy protection and covert communication (Ning.Y.X,2019).

To meet the demands of diversity of video application and the high trend of definition about the video compression performance requirements, high efficiency video coding (HEVC) is jointly developed by the International Telecommunication Union (ITU) and the Motion Picture Expert Group (MPEG). Based on H.264/AVC, it improves the compression of the performance and develops the new coding technologies. Therefore it can be better applied to the development of high-definition and ultra-high-definition video (Sullivan.G.J,Ohm.J.R\&Han.W.J,2012). Consequently, HEVC video information hiding technology has become a research hot spot.

HEVC video information hiding technology uses redundant information of video signal to hide secret information in the carrier. According to the different modification positions when the information is been embedded, the algorithms can be divided into four categories, i.e., prediction mode based modification, DST/DCT coefficient based modification, motion vector based modification and entropy coding based modification.

Chang et al. (2014) proposed a DCT / DST based information hiding algorithm for the first time, which effectively controlled the intra distortion drift; Liu et al. (2018) proposes a readable data 
hiding algorithm, which embedded the message in the fast brightness and could effectively avoid the problems of intra frame distortion drift and maintain the video quality. Li et al. (2016) uses the motion vector space to modify the $\mathrm{N}$ motion vector components in CTU as the carrier embedding the information. Yang et al. (2018) proposed an information hiding algorithm to modify the motion vector in P frame. Guo et al. (2020) proposed a new video steganography algorithm based on motion vector, the motion trend based (MTB) mapping strategy establish between motion vector and binary bit stream, which ensured the minimum additional distortion and steganography security.

This paper mainly discusses the problem of modifying the prediction model in the research of information hiding algorithm. By using the probability distribution of the optimal and suboptimal prediction models, the mapping rules are established between the prediction model and the secret information. In the research of h.264/AVC information hiding algorithm for intra prediction mode, $\mathrm{Hu}$ et al. (2008) firstly proposed the idea to modify the intra prediction mode to embed the secret information, and completed the information embedding by establishing the mapping relationship between the prediction mode and the secret information. Wang et al. (2010) according to the rate distortion generation value in the process of mode modulation. At the same time, the secret information can be embedded in the coding mode of I frame, P frame and B frame modulation macroblock, and it also can be optimized after the process of mode adjustment. Xu et al. (2011) according to the mapping relationship between secret information and prediction mode, the optimal prediction mode of modulation is the prediction mode with the same parity as the secret information and the minimum rate distortion generation value, which purpose is to complete the information embedding. Information hiding algorithm based on HEVC standard, Wang et al. (2014), reduces the errors caused by coding, prediction mode modulation is applied to intra luminance blocks with complex texture. According to the local texture feature (LBP), the algorithm searches all prediction modes that need parity mapping, and uses Lagrange rate distortion rate to modify the original prediction mode to the optimal prediction mode to achieve information hiding with the minimum cost. Xu et al. (2015), the differences of 33 directional prediction models are used to establish the mapping relationship between the difference of the prediction model and the secret information, and to complete the embedding of the secret information through the modulation of the prediction model. Dong et al. (2017), according to the intra coding process of HEVC and the distortion introduced by the modified pus coding, a steganographic channel model SCM (steganographic channel model) is established, the distortion optimization method is applied to the coding efficiency for the first time, and a new HEVC video steganography algorithm is obtained. Sibaji.G, et al. (2020), an anti-recompression watermarking algorithm based on intra-frame prediction mode is proposed. By analyzing the spatial texture of the video, the algorithm selects the reasonable candidate blocks by using the number of non-zero transform coefficients and the stability of the brightness prediction block. During the grouping process, the mode change of recompression is still completed in the same group, so as to realize the embedding of 2-bit information. Wang et al. (2019) firstly analyzed the probability distribution of all $4 \times 4$ intra prediction modes, and proposed a carrier selection method combining CU (coding black) and PU coding information to improve the security of steganographic video. In addition, matrix coding is used to encrypt the video. Zhong et al. (2021) proposed a Generative Adversarial Network (GAN)based intra prediction approach to enhance intra prediction accuracy. Gan's good training generator acts as the mapping from the adjacent reconstructed signal to the PU, and is applied to the encoder and decoder to reduce the bit rate in the experimental results.

In this paper, we propose a more effective encryption framework for the secret information embedding. Compared with the previous paper (Wang.J.J,Wang.R.D\&Li.W, 2014), the new contribution is mainly reflected in improving the embedding capacity without affecting the video quality. This paper presents an information hiding algorithm based on HEVC intra-frame prediction mode and matrix coding. By selecting 8 prediction modes for every 8 continuous luminance blocks in the frame, the hidden information is embedded. For the first 7 prediction modes, 3 bits of secret information is embedded through Hamming code parity verification matrix. As for the last prediction 
model, the least bit is modulated by the LSB algorithm to complete the embedding of 1 bit secret information. The algorithm has little influence on video quality and bit rate, and the decoding process is relatively easy. However, the algorithm has a certain degree of vulnerability. Under external attacks, the performance of steganography will be reduced.

The rest of the paper is organized as follows: the intra prediction of HEVC is explained in Section 2 and the description of the HEVC information hiding algorithm is showed in Section 3. In section 4, experiments and analysis are presented. Finally, the paper is concluded in Section 5.

\section{INTRA PREDICTION OF HEVC}

The coding framework used by HEVC is similar to that of the H.264/AVC standard, and it also belongs to the block-based hybrid coding structure (Shen. Y.F., Li, J.T., \&Zhu, Z.M. 2013). Compared with H.264/AVC, HEVC proposes a new technology in the prediction unit to improve efficiency of coding. Instead of using the macroblock partitioning mode in H.264/AVC, HEVC adopts the quadtree structure partitioning mode.

\subsection{Intra Prediction}

HEVC uses block-based multiparty prediction in frame prediction to eliminate the spatial correlation of images. There are 9 intra prediction modes in H.264/AVC, and they are refined into 35 prediction modes in HEVC, as shown in Figure 1. Among them, 2-34 represents 33 Angle prediction modes. 1 represents Planar mode, which is suitable for large flat areas. 0 represents the direct current (DC) mode and is suitable for areas where pixel values change slowly. The refined intra-frame prediction model makes the process of the video coding more efficient and the prediction more accurate.

Figure 1. 35 intra prediction modes for HEVC (Wan.S\&Yang.F.Z,2014)

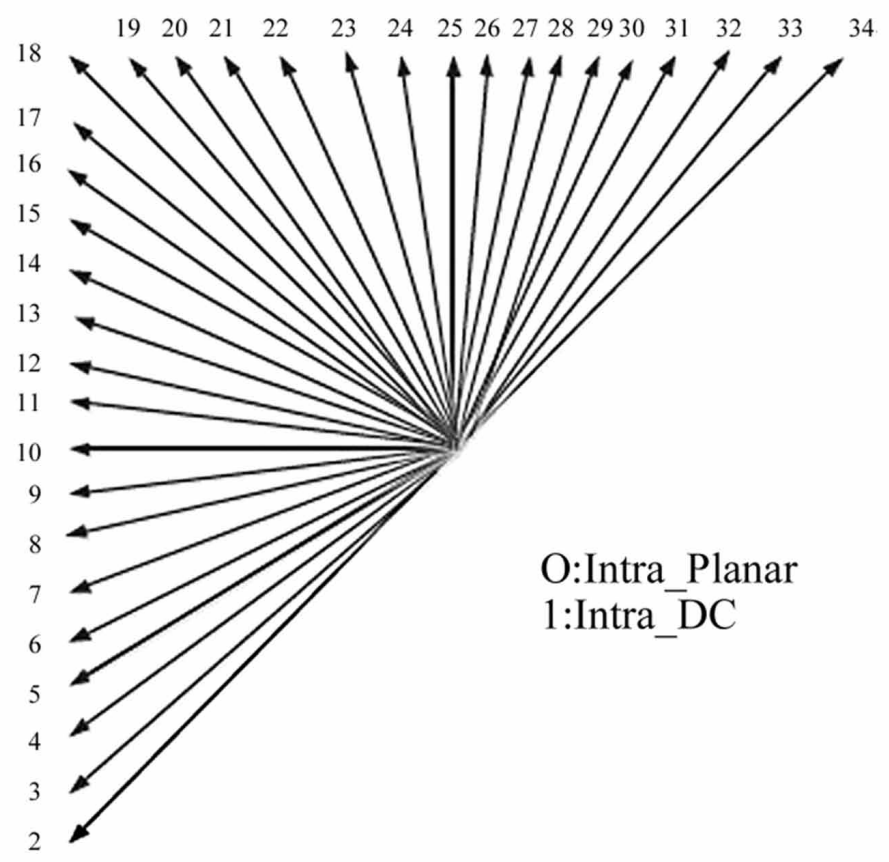


Because the adjacent pixels in the spatial domain of video signal have a strong correlation, the information redundancy between adjacent pixels can be reduced by using pixel prediction so as to be understood. As shown in Figure 2, there is a total of 16 brightness blocks to be coded in the intraframe prediction mode, denoted as $a \sim p$, and the predicted reference pixel is $A \sim M$. According to the pixel values of the upper and the prediction of left pixels, the current pixel value is obtained through the corresponding weighted average calculation (Wan.S\&Yang.F.Z,2014).

Figure 2. $4 \times 4$ intra prediction model of H.265/HEVC(Wan.S\&Yang.F.Z,2014)

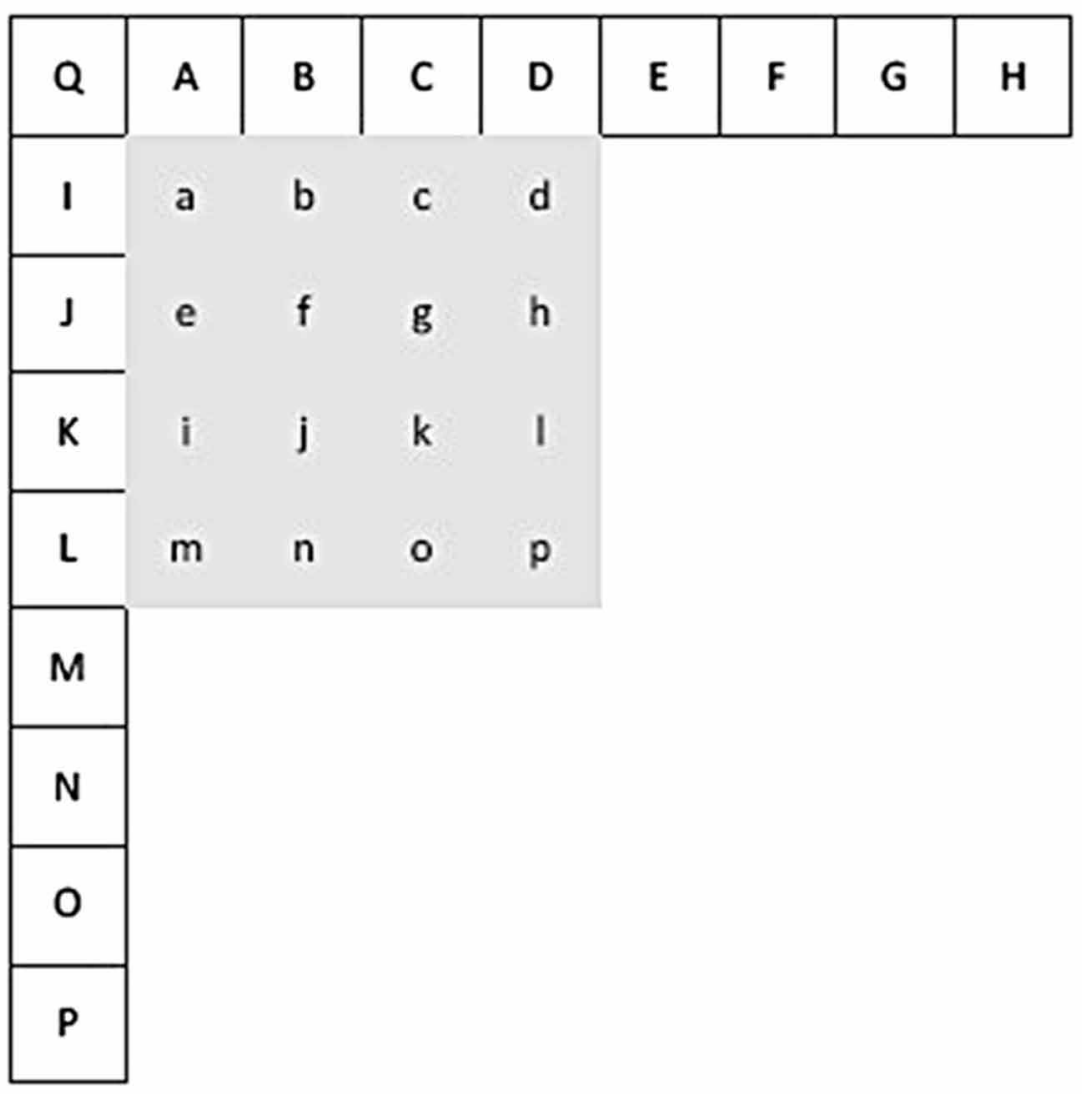

\subsection{Selection of Intra Prediction Mode}

The basic idea of intra-frame prediction is to use the pixel value of the encoded block in the current image to generate the predicted value of the current block. After the predicted value of the current block is generated, it is subtracted from the original pixel value of the current block to obtain the residual value, and then the residual value is transformed, quantized and entropy coded. The basic process of intra prediction is as follows: 
Firstly, all intra prediction modes are traversed to get the residual signal in each mode. Then, Hadamard transform is performed on the residual signal to calculate the SATD value of each mode. The rate distortion cost of each intra prediction mode is calculated by using the SATD value. Moreover several modes with the lowest rate distortion cost are selected as the prediction mode candidate set(Lainema J, Bossen.F\& Han. W,2012).

Then, the prediction mode used by the adjacent blocks that have been coded is added to the candidate set, and all the modes in the candidate set are traversed. The rate distortion cost value of the corresponding prediction mode is determined by using the Lagrange rate mode. The prediction mode with the lowest rate-distortion cost is selected as the best prediction mode of current prediction block.

\section{PROPOSED INFORMATIOM HIDING TECHNIQUE}

\subsection{Principle of Information Hiding}

Zhang et al.(2007) proposed the Hamming code +1 algorithm, which integrates the idea of LSB algorithm on the basis of matrix coding to realize the embedding of hidden information. Hamming code is a kind of linear debugging code, which inserts verification code into the transmitted information stream to detect and correct data bits.

In general, if the hamming code length is $n$, the number of information bit is $m$, and the check information length is $k$, the Hamming code can be denoted as:

$$
(n, m)=\left(2^{k}, 2^{k}-k-1\right)
$$

Here, the Hamming code $\boldsymbol{H}(7,4)$ with parameters $k=3$ is used here, which can be expressed as:

$$
H=\left[\begin{array}{lllllll}
0 & 0 & 0 & 1 & 1 & 1 & 1 \\
0 & 1 & 1 & 0 & 0 & 1 & 1 \\
1 & 0 & 1 & 0 & 1 & 0 & 1
\end{array}\right]
$$

Matrix encoding is used to modify the LSB bit of a data in the obtained host vector $a=\left(a_{1}, a_{2}, \ldots, a_{2^{k}-1}\right)$ and embed secret information on the host vector through the parity verification matrix of Hamming code $\boldsymbol{H}$. The embedding method is as follows:

$$
\begin{aligned}
& \operatorname{LSB}(a)=\left(\operatorname{LSB}\left(a_{1}\right), \operatorname{LSB}\left(a_{2}\right), \ldots, \operatorname{LSB}\left(a_{2^{k}-1}\right)\right), \\
& l^{T}=\left(l_{1}, l_{2}, \ldots, l_{j}\right)^{T}=H^{\prime \prime}(\operatorname{LSB}(a))^{T},
\end{aligned}
$$

where $\operatorname{LSB}(a)$ represents the least-significant extraction operation for the data of host vector $a, l$ represents the check vector, $l_{j} \in(0,1)$, the length is $k, 1 \leq j \leq k$, and ()$^{T}$ represents the transpose of the matrix.

To illustrate the embedding principle, assuming that there being the embedded 4-bit information is $x=\left(x_{1}, x_{2}, x_{3}, x_{4}\right)$, First, a matrix encoding operation is adopted for the first 7 host data 
$\left(a_{1}, a_{2}, a_{3}, a_{4}, a_{5}, a_{6}, a_{7}\right)$. The difference vector is computed by $d^{T}=l^{T} \oplus x^{T}$ and $d^{T}$ is converted to the corresponding decimal, where $\oplus$ represents the XOR operator. Finally, according to Eq. (4) to determine the relationship between $m_{4}$ and the 4 th secret information $x_{4}$, and then whether the 8th host data needs to be modified is decided.

$m_{4}=\left(\frac{a_{1}}{2}+\frac{a_{2}}{2}+\ldots+\frac{a_{7}}{2}+a_{8}\right) \bmod 2$

where $\lfloor\cdot\rfloor$ represents round down. The specific embedding rules are as follows:

Rule 1: If $d^{T}=0$, the first 7 bits of host data do not need to be modified. When $m_{4} \neq x_{4}$, modify the lowest level of the eighth host data, and perform +1 operation. If $m_{4}=x_{4}$, no modification is required.

Rule 2: If $d^{T} \neq 0$, the first 7 bits of host data need to be modified. The decimal value of $d^{T}$ is the data corresponding to the ordinal value $a_{i}$ of the first seven host data. If $m_{4} \neq x_{4}$, modify the lowest level of the eighth host data, and perform +1 operation. If $m_{4}=x_{4}$, no modification is required.

Through the introduction above, on the basis of the original research, this paper uses matrix coding combined with LSB algorithm to improve the embedding capacity of secret information and to meet the requirements of video steganography.

\subsection{The Embedding Process of Secret Information}

The algorithm proposed in this paper can embed 4-bit secret information in 8 consecutive $4 \times 4$ luminance blocks through matrix encoding and LSB modification. The specific steps are as follows:

Step 1: The prediction modes of 8 continuous $4 \times 4$ luminance blocks are selected as the host data vector $\left(a_{1}, a_{2}, a_{3}, a_{4}, a_{5}, a_{6}, a_{7}, a_{8}\right)$ for intra-frame prediction. In the process of information embedding, because the lowest bit is involved, the rate-distortion model is combined with the original prediction mode to select the intra prediction mode with the least cost as the sub-optimal mode (Liu, J.M. 2014).

Step 2: Get the check vector $l^{T}$ through Eq. (3), calculate the difference vector $d^{T}$, and convert it to the corresponding decimal value.

Step 3: Get $m_{4}$ according to Eq. (4). If $d^{T}=0$, the secret information is embedded according to the modification rule 1 in Section 3.1. Otherwise, the secret information is embedded according to the modification rule 2 . The host vector containing the secret information is obtained and encoded.

Step 4: Repeat the above operation until the end of embedding.

Assuming that the prediction modes of the 8 luminance $4 \times 4$ blocks selected in the prediction block are $2,7,10,12,5,18,30,24$, and the secret information $x=(0,1,0,1)$, then the host vector is $a=(2,7,10,12,5,18,30,24)$ and the check vector is $l^{T}=H^{\prime \prime}(\operatorname{LSB}(a))^{T}=(1,1,1)^{T} \cdot d^{T}$ 
is calculated as $d^{T}=l^{T} \oplus x^{T}=(1,0,1)^{T}=5$. Therefore, it is necessary to modify the 5 th host data, which can be obtained $m_{4}=1=x_{4}$ according to Eq. (4). The host vector containing secret information $a^{\prime}=(2,7,10,12,6,18,30,25)$ can be obtained according to the modification rules.

\subsection{THE EXTRACTION PROCESS OF HIDDEN INFORMATION}

The extraction of hidden information is simple and fast. The hidden information can be extracted only by partially decoding I frames. The specific steps are as follows:

Step 1: Determining whether the encoding mode of the current luminance block is the $4 \times 4$ encoding mode in intra prediction. If not, then no secret information is extracted from the current block and step 3 is performed. If the encoding mode of the current block is $4 \times 4$ encoding mode, decode the prediction mode of 8 consecutive $4 \times 4$ intra-frame luminance blocks including this $4 \times 4$ block.

Step 2: The host vector $a^{\prime}=\left(a_{1}^{\prime}, a_{2}^{\prime}, a_{3}^{\prime}, a_{4}^{\prime}, a_{5}^{\prime}, a_{6}^{\prime}, a_{7}^{\prime}, a_{8}^{\prime}\right)$ containing hidden information is obtained from the decoded prediction modes of 8 consecutive $4 \times 4$ blocks. The check vector $l^{T}$ is calculated by Eq. (3), and $l$ is the hidden information. Get $m_{4}^{\prime}$ according to Eq. (4), i.e., the 4 th secret information $x_{4}$ is obtained. Then the secret information $x=\left(x_{1}, x_{2}, x_{3}, x_{4}\right)$ is obtained.

Step 3: Repeat the above operation for each subsequent 8 consecutive $4 \times 4$ blocks until the hidden information is extracted or the video sequence is decoded.

Assuming that the host vector containing hidden information obtained at the decoding end is $(2,7,10,12,6,18,30,25)$, the check matrix $l^{T}=H^{\prime \prime}\left(\operatorname{LSB}\left(a^{\prime}\right)\right)^{T}=(0,1,0)^{T}$ is calculated first. $l$ is the is the first 3 bits of information, i.e., $\left(x_{1}, x_{2}, x_{3}\right)$. Then according to Eq. (4), $m_{4}=\left(\frac{a_{1}}{2}+\frac{a_{2}}{2}+\ldots+\frac{a_{7}}{2}+a_{8}\right) \bmod 2=1$, namely $x_{4}=1$.So the extracted hidden information is $x=(0,1,0,1)$.

\section{EXPERIMENTAL RESULTS AND ANALYSIS}

The algorithm is implemented on the HEVC reference software model HM16.16. Five video sequences with different resolutions (i.e., BasketballDrill_832 4480 ,Videyo_3_1280×720,Videyo_4_ $1280 \times 720$,Jonhnny_1280 $\times 720$ and BQmall_832 $\times 480$ ) are used to test the algorithm performance. Table 1 shows the main coding configuration parameters of the reference software, and the other parameters are configured by default.

\subsection{Subjective Video Quality Analysis}

Figure 3 shows the video frames before and after embedding the secret information when $Q P=26$. It can be seen from the figure that there is no obvious distortion in the video frame before and after embedding the secret information, the algorithm only modifies the prediction mode, it does not affect the following processes such as quantization transformation (inverse quantization and inverse change), filtering and entropy coding, which indicates that the algorithm has a good imperceptibility. 
Table 1. Configuration parameters of the HM

\begin{tabular}{|l|l|}
\hline Parameter & Configuration \\
\hline Frames to be encoded & 100 \\
\hline Frame rate/fps & 30 \\
\hline Intra period & 1 \\
\hline GOP Size & 1 \\
\hline RDOQTS & 1 \\
\hline
\end{tabular}

Figure 3. Comparison of the visual quality between the reconstructed frames before and after embedding the secret information

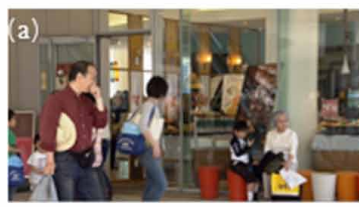

(a) BQmall_832 $\times 480(39.9302 \mathrm{~dB})$

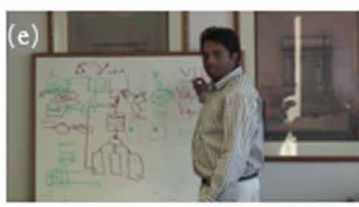

(d)Videyo_3_1280 $\times 720(43.509 \mathrm{~d}$

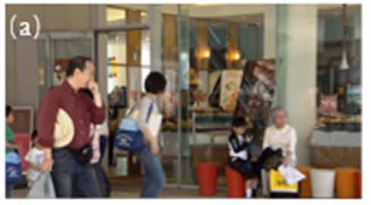

(a) BQmall $\sim 832 \times 480(39.8733 \mathrm{~dB})$

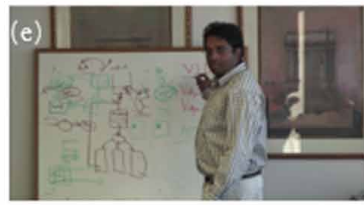

(d)Videyo_3_1280 $\times 720(43.4715 \mathrm{~dB})$

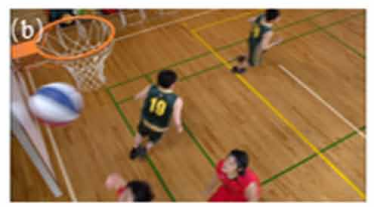

(b) BasketballDrill_ $832 \times 480(39.7592 \mathrm{~dB})$

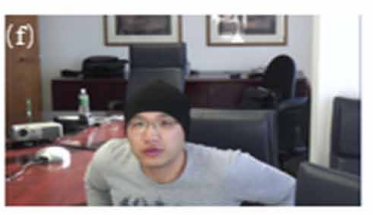

(e)Videyo_4_1280 $\times 720(43.2725 \mathrm{~dB})$

(i) Original reconstructed frames

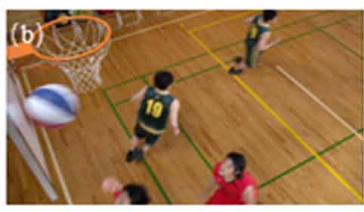

(b) BasketballiDrill_ $832 \times 480(39.7210 \mathrm{~dB})$

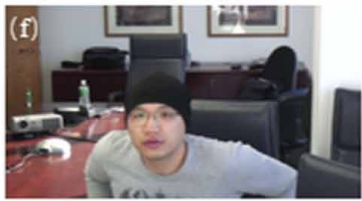

(e)Vidy._4_1280 × $720(43.2397 \mathrm{~dB})$

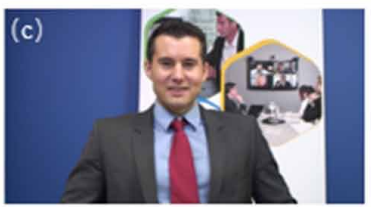

(c) Jonhnny_1280 $\times 720(43.3638 \mathrm{~dB})$

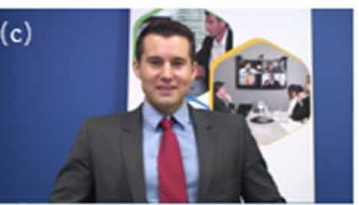

(c) Jonhnny_ $1280 \times 720(43.3398 \mathrm{~dB})$

(ii) Marked reconstructed frames(with the hiding information)

\subsection{Objective Video Quality Analysis}

For the performance evaluation of the algorithm, it is necessary to further analyze the objective quality of the video, which mainly being considered the PSNR (Xu.D.W,Wang.R.D,\&Shi.Y.Q,2014), SSIMs (Wang.Z,Bovik.A.C\&Sheikh,.H.R,2004)and other factors. $Q P$ is set to 26. PSNR is an 
important evaluation index used to represent the difference between the original video sequence and the embedded video sequence, $P S N R$ is defined as:

$$
P S N R=10 \times \log _{10}\left(\frac{M A X_{A}^{2}}{M S E}\right)(d B),
$$

Figure 4 shows the bar graph of the $P S N R$ values before and after embedding the hidden information. It can be seen from the figure that the overall $P S N R$ value before and after embedding information has little change, and there is no significant difference. $\triangle P S N R$ is within the range of $-0.02--0.06 \mathrm{~dB}$, with an average value of $-0.04 \mathrm{~dB}$. $\triangle P S N R$ is defined as:

Figure 4. Comparison of the PSNR before and after embedding the hidden information

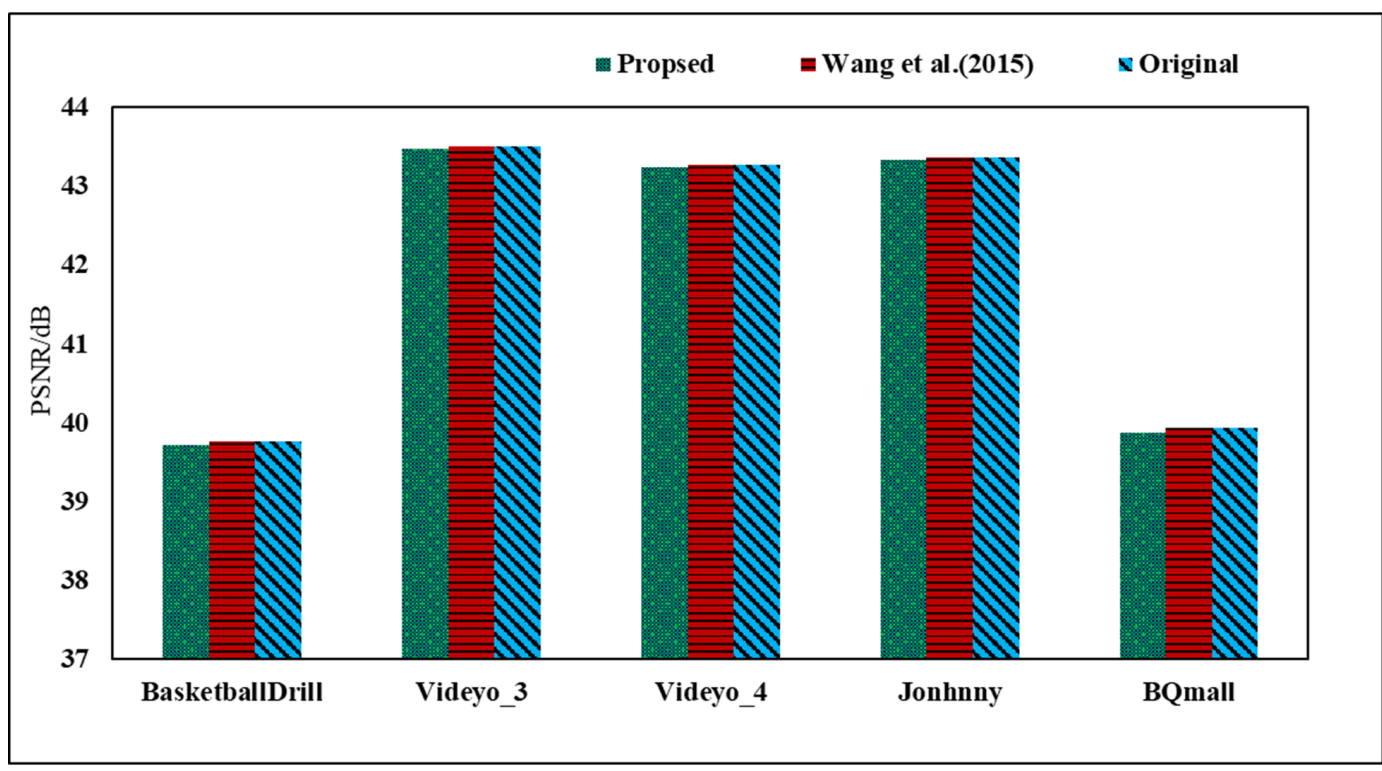

$\triangle P S N R=P S N R^{\prime}-P S N R$

Where $\triangle P S N R$ represents the difference of $P S N R$ before and after embedding the hidden information, $P S N R$ represents the value before embedding information, and $P S N R^{\prime}$ represents the value after embedding information.

The evaluation index of $S S I M$ can measure the similarity of two images within the range of $0-1$. The larger the value is, the higher the similarity is. As it could also be seen from Figure 5, the values of SSIM the test sequences before and after embedding private information are all above 0.945 , which was compared with the algorithm in literature (Wang.J.J,Wang.R.D\&Li.W,2014) and the original video, indicating that the embedded information had little impact on the video quality. 
Figure 5. Comparison test results ( $S S I M$ )

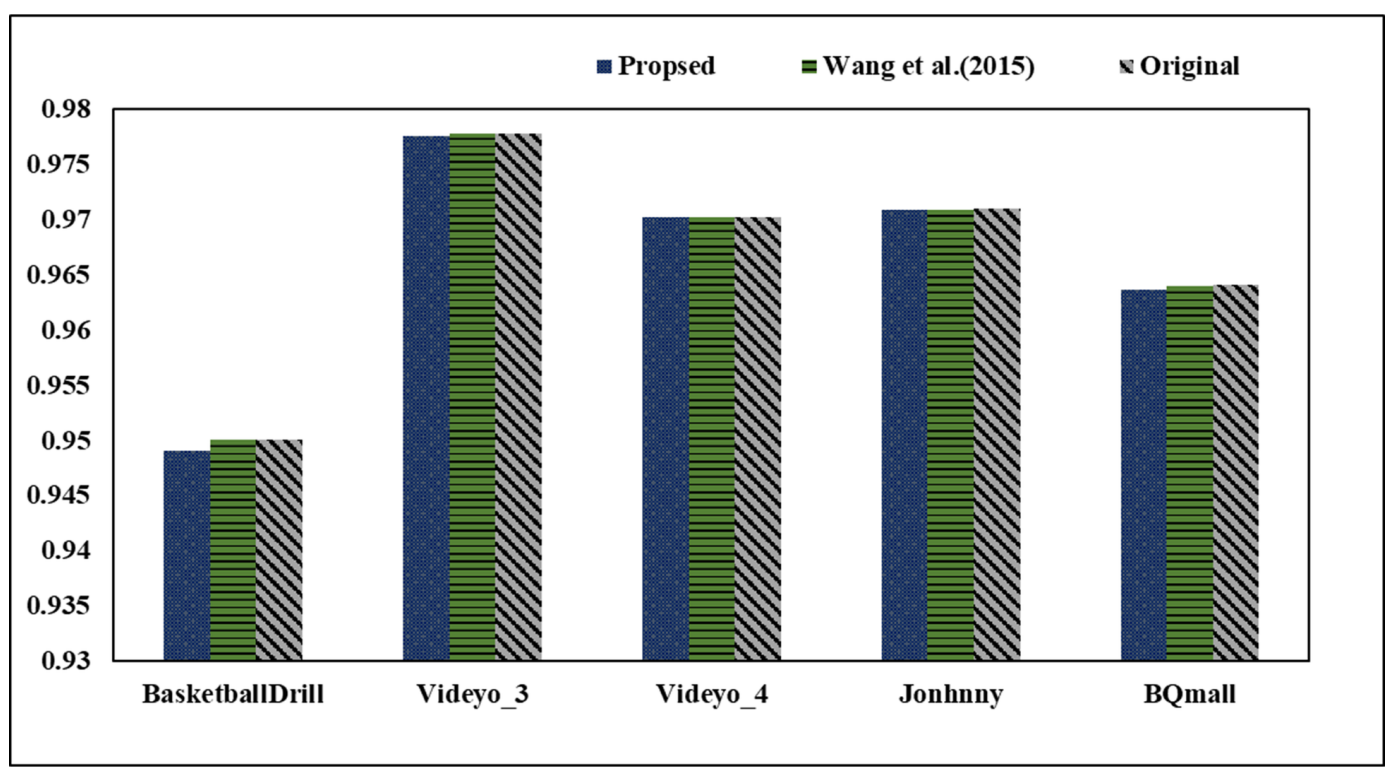

\subsection{Bit Rate Variation Analysis}

Figure 6 shows the $B R I$ value before and after embedding hidden information, where $B R I$ is defined as:

Figure 6. $B R I$ test results

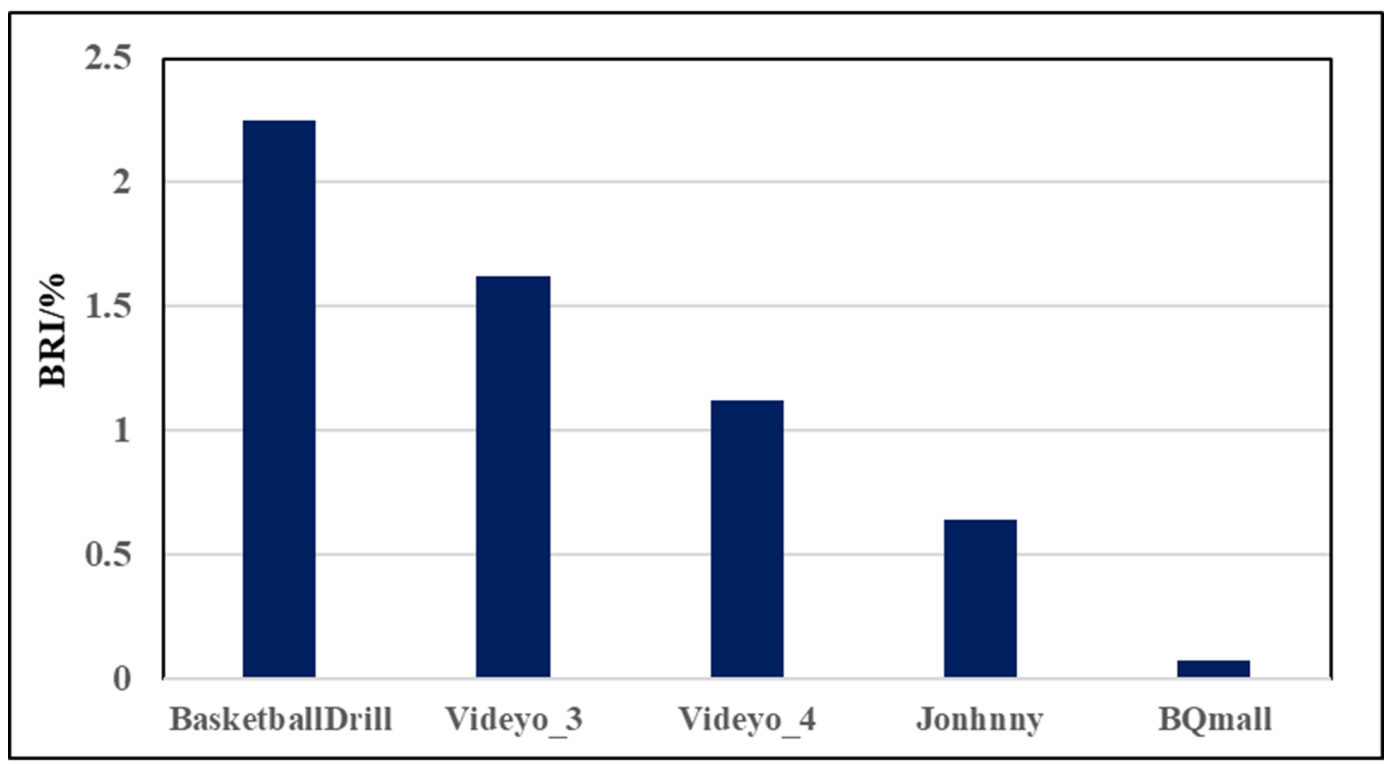


$B R I=\frac{R^{\prime}-R}{R} \times 100 \%$,

where $R$ represents the bit rate of the video before embedding information, $R^{\prime}$ represents the bit rate of the video after embedding information. Because of the modification of prediction mode in the process of information hiding, generally speaking, this change will result in the increase of compressed code $B R I$. The bit rate of the five videos increased by $1.14 \%$ on average.

\subsection{Performance Analysis and Comparison}

Table 2 shows the test results of different test sequences when $\mathrm{QP}=26$. By analyzing the data in the

Table 2. Experimental results of the comprehensive performance(QP=26)

\begin{tabular}{|l|l|l|l|}
\hline Sequence & $B R I / \%$ & $\triangle P S N R / \mathbf{d B}$ & Capacity/bit \\
\hline BasketballDrill & 2.25 & -0.0382 & 20146 \\
\hline Videyo_3 & 1.62 & -0.0375 & 13029 \\
\hline Videyo_4 & 1.12 & -0.0328 & 7325 \\
\hline Jonhnny & 0.64 & -0.0240 & 10272 \\
\hline BQmall & 0.071 & -0.0569 & 31085 \\
\hline
\end{tabular}

table, the following conclusions can be drawn:

- The fluctuation of PSNR and bit rate is not only caused by the modification of intra prediction mode, but also caused by the deviation of prediction of adjacent blocks, which leads to intra frame distortion drift.

-Different video test sequences have different embedding capacity mainly because of different resolution and video content. Therefore, the number of $4 \times 4$ luminance blocks that meet the embedding conditions is not consistent, which leads to the great differences in the number of embedded hidden information.

-For different video sequences with the same resolution (such as BasketballDrill_832 8480 and BQmall_ $832 \times 480$ ), because of the different video content, the number of $4 \times 4$ intra-frame luminance blocks that meet the conditions is also inconsistent, resulting in difference in embedding capacity.

-The value of PSNR before and after embedding the hidden information changes very little and has little influence on video quality, which can satisfy the visual imperceptibility of video information hiding algorithm.

Table 3 shows the performance comparison of PSNR, SSIM and embedding capacity between the algorithm mentioned in (Wang.J.J,Wang.R.D\&Li.W,2014) and the proposal of algorithm when $Q P=26$. The data shows that the $P S N R$ value of this algorithm decreases, mainly due to the increase in embedding capacity. The increase of embedded information leads to the increase of the modification quantity of the intra-frame prediction mode during the encoding process of the video, so that the video quality decreases. But the SSIM value is still above 0.945 . 
Table 3. Performance comparison $(Q P=26)$

\begin{tabular}{|c|c|c|c|c|}
\hline Sequence & & $P S N R$ /dB & $S S I M$ & Capacity /bit \\
\hline & Proposed & 39.7210 & 0.9491 & 20146 \\
\hline \multirow[t]{3}{*}{ BasketballDrill } & Wang et al. (2015) & 39.7587 & 0.9501 & 16681 \\
\hline & Original & 39.7592 & 0.9501 & \\
\hline & Proposed & 43.4715 & 0.9776 & 13029 \\
\hline \multirow[t]{3}{*}{ Videyo_3 } & Wang et al. (2015) & 43.5087 & 0.9778 & 10450 \\
\hline & Original & 43.5090 & 0.9778 & \\
\hline & Proposed & 43.2397 & 0.9702 & 7325 \\
\hline \multirow[t]{3}{*}{ Videyo_4 } & Wang et al. (2015) & 43.2723 & 0.9702 & 6952 \\
\hline & Original & 43.2725 & 0.9703 & \\
\hline & Proposed & 43.3398 & 0.9709 & 10272 \\
\hline \multirow[t]{3}{*}{ Jonhnny } & Wang et al. (2015) & 43.3636 & 0.9709 & 8293 \\
\hline & Original & 43.3638 & 0.9710 & \\
\hline & Proposed & 39.8733 & 0.9637 & 31085 \\
\hline \multirow[t]{2}{*}{ BQmall } & Wang et al. (2015) & 39.9294 & 0.9640 & 22711 \\
\hline & Original & 39.9302 & 0.9641 & \\
\hline
\end{tabular}

Fig.7 shows the comparison graph of the embedding capacity between the algorithm in (Wang.J.J,Wang.R.D\&Li.W,2014) and the proposed algorithm. The results of the test show that the algorithm improves the embedding capacity to a certain extent.

Figure 7. Embedded capacity comparison test results

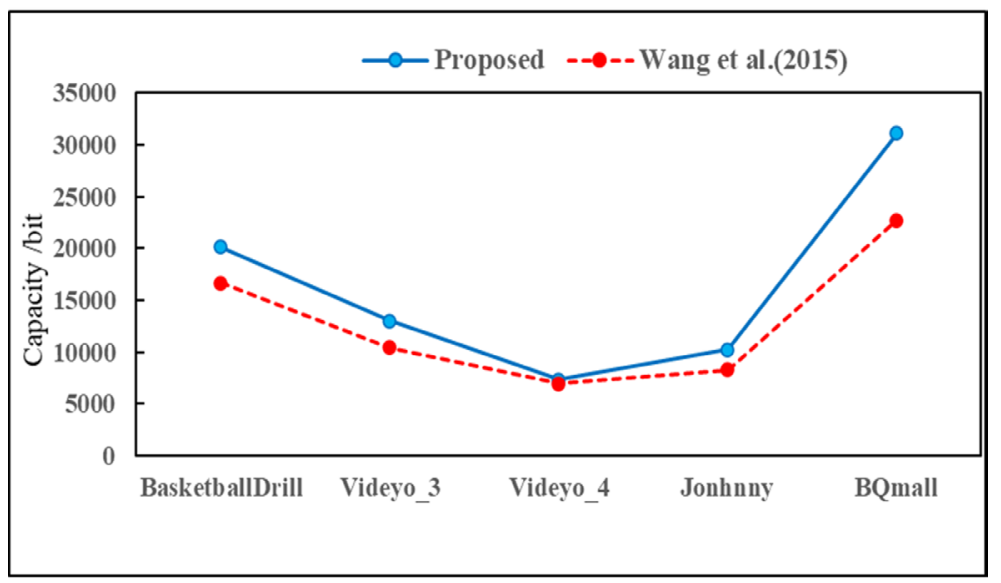




\section{CONCLUSION}

This paper proposes an information hiding algorithm based on HEVC intra prediction mode and matrix coding, which embeds the secret information by modifying the prediction mode. The algorithm ensures the embedding capacity of the information. Because of the increase of the embedding capacity, the bit error rate is increased, so the extracted information and the original information have some errors when the encoded video sequence is subjected to re compression attack, noise attack or filtering attack. In the field of digital crime and forensics, this method can be used for the preliminary analysis of digital video tampering forensics even if the proposed hiding algorithm is fragile. To solve this problem, the future research will be discussed on how to achieve the robustness of the algorithm when the prediction mode is modified.

\section{ACKNOWLEDGMENT}

This work is supported by the National Natural Science Foundation of China (61771270, 62071267); Zhejiang Provincial Natural Science Foundation of China (LR20F020001). 


\section{REFERENCES}

Chang, P. C., Chung, K. L., Chen, J. J., Lin, C. H., \& Lin, T. J. (2014, February). A DCT/DST-based error propagation-free data hiding algorithm for HEVC intra-coded frames. Journal of Visual Communication and Image Representation, 25(2), 239-253. doi:10.1016/j.jvcir.2013.10.007

Dong, Y., Jiang, X. H., Sun, T. F., \& Xu, D. W. (2017, August). Coding Efficiency Preserving Steganography Based on HEVC Steganographic Channel Model. Digital Forensics and Watermarking, 10431, 149-162. doi:10.1007/978-3-319-64185-0_12

Guo, M. Y., Sun, T. F., Jiang, X. H., Dong, Y., \& Xu, K. (2020, August). A Motion Vector-Based Steganographic Algorithm for HEVC with MTB Mapping Strategy. Digital Forensics and Watermarking, 12022, 293-306. doi:10.1007/978-3-030-43575-2_25

Hu, Y., Zhang, C. T., \& Su, Y. T. (2008). An information hiding algorithm based on H.264/AVC. Journal of Electronics (China), 36(4), 690-694.

Lainema, J., Bossen, F., Han, W., Min, J., \& Ugur, K. (2012, December). Intra coding of the HEVC standard. IEEE Transactions on Circuits and Systems for Video Technology, 22(12), 1697-1706. doi:10.1109/ TCSVT.2012.2221525

Li, S. B., Wang, L. R., Liu, P., \& Huang, Y. F. (2016, July). A HEVC Information Hiding Approach Based on Motion Vection Space Encoding. Chinese Journal of Computer, 39(7), 1450-1463.

Liu, J. M. (2014). Research on video information hiding algorithm based on H.264 prediction mode (Master's thesis). Southwest Jiaotong University.

Liu, Y. X., Liu, S. Y., Zhao, H. G., \& Liu, S. (2018, July). A new data hiding method for H.265/HEVC video streams without intra-frame distortion drift. Multimedia Tools and Applications, 78(6), 6459-6486. doi:10.1007/ s11042-018-6320-y

Mohanarathinam, A., Kamalraj, S., \& G, K. D. (2019, September). Prasanna Venkatesan, et al. Digital watermarking techniques for image security: a review. Journal of Ambient Intelligence and Humanized Computing, $11(8), 3221-3229$.

Ning, Y. X. (2019). Research on video based information hiding algorithm (Master's thesis). Qingdao University.

Shen, Y. F., Li, J. T., Zhu, Z. M., \& Zhang, Y.-D. (2013, November). High Efficiency Video Coding. Chinese Journal of Computers, 36(11), 2340-2355. doi:10.3724/SP.J.1016.2013.02340

Sibaji, G., Arijit, S., \& Prabin, K. B. (2020, July). Prediction mode based H.265/HEVC video watermarking resisting re-compression attack. Multimedia Tools and Applications, 79(25-26), 18089-18119. doi:10.1007/ s11042-019-08301-w

Sullivan, G. J., Ohm, J. R., Han, W. J., \& Wiegand, T. (2012). Overview of the High Efficiency Video Coding (HEVC) Standard. IEEE Transactions on Circuits and Systems for Video Technology, 22(12), 1649-1668. doi:10.1109/TCSVT.2012.2221191

Wan, S., \&Yang, F.Z. (2014). A new generation of high efficient video coding standard-H.265/HEVC: principle and implementation. Peking: Publishing House of Electronics Industry.

Wang, J., Jia, X. Q., Kang, X. G., \& Shi, Y. Q. (2019, December). A Cover Selection HEVC Video Steganography Based on Intra Prediction Mode. IEEE Access: Practical Innovations, Open Solutions, 7, 119393-119402. doi:10.1109/ACCESS.2019.2936614

Wang, J. J., Wang, R. D., \&Li, W. (2014, August). An information hiding algorithm for HEVC based on intra prediction. Journal of Optoelectronics Laser, 25(8), 1578-1585.

Wang, R. D., Zhu, H. L., \& Xu, D. W. (2010, May). An information hiding algorithm for H.264/AVC based on encoding mode. Opto-Electornic Engineering, 37(5), 144-150.

Wang, Z., Bovik, A. C., Sheikh, H. R., \& Simoncelli, E. P. (2004, December). Image quality assessment: From error visibility to structural similarity. IEEE Transactions on Image Processing, 13(4), 600-612. doi:10.1109/ TIP.2003.819861 PMID:15376593 
Xu, D. W., \& Wang, R. D. (2011, November). An improved information hiding algorithm with prediction mode for H.264/AVC. Opto-Electornic Engineering, 38(11), 99-105.

Xu, D. W., Wang, R. D., \& Shi, Y. Q. (2014, April). Data Hiding in Encrypted H.264/AVC Video Streams by Codeword Substitution. IEEE Transactions on Information Forensics and Security, 9(4), 596-606. doi:10.1109/ TIFS.2014.2302899

Xu, J., Wang, R.D., \& Huang, M.L. (2015, September). A date hiding algorithm for HEVC based on the differences of intra prediction modes. Journal of Optoelectronics Laser, 26(9), 1753-1760.

Yang, J., Li, S. B., \& Deng, H. J. (2018). An efficient information hiding method based on motion vector space encoding for HEVC. Multimedia Tools and Applications, 77(10), 11979-12001. doi:10.1007/s11042-017-4844-1

Zhang, W., Wang, S., \& Zhang, X. (2007, August). Improving embedding efficiency of covering codes for applications in steganography. IEEE Communications Letters, 11(8), 680-682. doi:10.1109/LCOMM.2007.070438

Zhong, G. Y., Wang, J., Hu, J. Y., \& Liang, F. (2021, January). A GAN-Based Video Intra Coding. Electronics (Basel), 10(2), 132-132. doi:10.3390/electronics10020132

Yong Liu is a graduate student at School of mathematics and big data, Anhui University of Science and Technology, China. His research interests include digital watermarking and information hiding.

Dawen Xu received the M.S. degree in communication and information system from NingBo University, China, in 2005 and the Ph.D degree in computer applied technology from Tongji University, China, in 2011. He is a visiting scholar at New Jersey Institute of Technology from August 2012 to August 2013. He is now a Professor at School of Electronics and Information Engineering, Ningbo University of Technology, China. His research interests mainly include digital watermarking and information hiding, signal processing in the encrypted domain. He has served as a technical paper reviewer for IEEE conferences, journals \& magazines. 